\title{
El combate por la paz de Jean Jaurès: análisis de sus textos en l'Humanité
}

\author{
Jean Jaurès' struggle for peace: a review of his articles \\ published in l'Humanité
}

\author{
Antonia Ceballos Cuadrado, Universidad de Cádiz, España \\ Av. Dr. Gómez Ulla, 1, 11003 Cádiz \\ antoniaceballos3@gmail.com | Orcid: https://orcid.org/0000-0003-4745-2285
}

DOI: http://dx.doi.org/10.12795/Ambitos.2019.i45.04

\begin{abstract}
Resumen
Jean Jaurès fue el parlamentario más joven de la Tercera República francesa, fue doctor en filosofía y profesor de esta materia, notable orador, fundador de la Sección Francesa de la Internacional Obrera (SFIO) que consumó la unión de los socialistas franceses; pero ante todo fue un gran periodista, fundador de un periódico que ha conseguido sobrevivir al siglo XX y lo que llevamos de XXI: I'Humanité.

Con el presente trabajo exploramos una constante en toda su obra: el combate por la paz, un combate teórico y práctico que le costó la vida en la víspera del estallido de la Primera Guerra Mundial a manos de un militante de la ultraderecha francesa. En concreto, analizamos las ideas que defiende en el periódico que creó desde su fundación hasta su asesinato.

El corpus utilizado es de 50 textos escogidos a través de las fuentes bibliográficas consultadas. Las herramientas del análisis del discurso, así como las teorías que
\end{abstract}


estudian la intertextualidad nos han servido para acercarnos a los textos y comprender mejor su significado filosófico, político y periodístico.

Una vez realizado el análisis, concluimos que podemos agrupar su defensa de la paz en cuatro ideas clave: la equivalencia semántica entre República, democracia y socialismo; la complementariedad entre la nación y el internacionalismo; unas relaciones internacionales basadas en el derecho y el arbitraje; y la importancia del deber y de la responsabilidad.

Jean Jaurès creyó hasta su último aliento que la paz era posible y un siglo después sus reflexiones siguen siendo útiles.

\section{Abstract}

Jean Jaurès was the youngest Member of Parliament of the Third French Republic, he was a doctor and professor of philosophy, prominent orator, founder of the French Section of the Workers' International (SFIO) that consummated the union of the French Socialists; nevertheless he was primarily a great journalist, founder of a newspaper, l'Humanité, that has managed to survive the $X X$ and $X X I$ century to date.

Through this paper, we examine a common theme in his life's work: the struggle for peace, a theoretical and practical struggle that cost him his life on the eve of the outbreak of the First World War in the hands of a militant of the French far-right movement. In particular, we analyze the ideas he defends in his newspaper from its foundation to his murder.

The corpus we used consists of 50 texts chosen from the consulted bibliographic sources. The tools of discourse analysis, as well as the theories that study intertextuality, have helped us to get closer to the texts and better understand their philosophical, political and journalistic meaning.

Upon completion of the analysis, we conclude that we can group its defense of peace into four key ideas: the semantic equivalence between the Republic, democracy and socialism; the complementarity between the nation and internationalism; international relations based on law and arbitration; and the importance of duty and responsibility.

Jean Jaurès believed until his last breath that peace was possible and a century later his reflections are still useful.

Palabras clave: Jean Jaurès, l'Humanité, Primera Guerra Mundial, paz, periodismo

Keywords: Jean Jaurès, l'Humanité, First World War, peace, journalism

\section{INTRODUCCIÓN}

La guerra ha acompañado al ser humano desde sus albores. Mirando a nuestro alrededor parece que hay un fracaso colectivo en la solución pacífica de los conflictos. Por eso, hemos escogido el pensamiento de paz de Jean Jaurès como objeto de 
estudio porque estamos de acuerdo con Kermoal (2014, p. 2) cuando afirma que "luchando contra la ley de los tres años y por la defensa de la paz, Jaurès nos da un método de trabajo que aplica permanentemente: antes de luchar contra, antes de actuar, hay que reflexionar, estudiar el problema, sumergirse en los meandros de la cuestión, sea cual sea la dificultad y la novedad. La acción emprendida entonces solo puede ser eficaz".

Jean Jaurès hizo prácticamente de todo: fue el diputado más joven de la Tercera República, doctor en filosofía, historiador, uno de los oradores más importantes de su época y, por supuesto, periodista. Su obra es inmensa e incluye los discursos en la Asamblea nacional, los discursos en las reuniones de la Internacional, sus intervenciones públicas, libros de filosofía y análisis históricos, miles de artículos periodísticos, entre otros.

Aquí analizamos su combate por la paz en los artículos de l'Humanité, el periódico que fundó el 18 de abril de 1904 y del que fue director político hasta el día de su asesinato a manos de Raoul Villain, un militante ultranacionalista, el 31 de julio de 1914, la víspera de la Primera Guerra Mundial.

Consideramos que es necesario estudiar este período de su obra porque "los diez últimos años de su vida, Jean Jaurès concentra su trabajo en la única cuestión de la guerra y de la paz" (Duclert, 2013, p.47). El corpus utilizado está compuesto por 50 textos publicados entre estas dos fechas (18 de abril de 1904 y 31 de agosto de 1914). Los textos han sido seleccionados siguiendo a los autores y autoras que mejor conocen la obra de Jaurès: Candar (2009), Duclert (2013), Fabre (2014 y 2015), Kermoal (2014, 2014b, 2014c y 2014d), Marcobelli (2013), Rebérioux (1994), Rioux (2005) y Silvestre (2010). Asimismo, se realiza una revisión crítica de las aportaciones de los autores y autoras antes mencionados, considerando las (re)significaciones de los textos de Jaurés al valorarse en su contexto periodístico.

De este modo, la aproximación a los textos se hace desde esta doble vertiente: valorando una fuerte componente filológica, en torno a las palabras guerra y paz, y una fuerte componente periodística, en relación con el modo en que Jaurés estructura su opinión en su contexto. Para comprender y desgranar el contenido de nuestro corpus hemos recurrido principalmente al análisis del discurso, la lingüística aplicada y las diferentes teorías de la intertextualidad buscando hallar en las interrelaciones entre el sujeto que opina y el contexto sociopolítico la construcción de la significación de su cosmovisión. ${ }^{2}$

Con este trabajo reivindicamos las contribuciones jauresianas al oficio y nos preguntamos con Silvestre: “¿Este periodismo visionario es aún un periodismo que puede "hablarnos" hoy en día?" (2010, p. 113). Intentamos demostrar que la respuesta es que sí. 


\section{JAURÈS, PERIODISTA}

En 1885, cuando tenía 26 años, Jean Jaurès se convierte en el diputado más joven de Francia. Antes de eso, en la región del Tarn habían leído ya sus artículos en L'Union Républicaine, Le Réveil Républicain, L'Avenir y Le Courrier. Pero si hay un periódico donde aprende a ser periodista ese es La Dépêche de Toulouse en el que escribió 1312 artículos desde 1887 hasta su asesinato y donde, incluso, se atrevió a crear un alter ego literario, le Liseur. Solo por esta obra, Jaurès merecería ser llamado "periodista" con mayúsculas, pero es que además hizo otras muchas e importantes contribuciones al oficio. Desde 1893 hasta 1903, colaboró en La Petite République llegando a ser incluso codirector de la publicación. En este periódico escribe Jaurès una serie de artículos sobre el asunto Dreyfus, "un documento capital para comprender el caso" (Silvestre, 2010, p. 47) con el que Jaurès "inaugura la historia ultra-contemporánea" (Rebérioux, 2010, p. 40). Jaurès escribió incluso en el conservador Le Matin; en este periódico, que criticará tan duramente en l'Humanité, nos dejó 43 artículos, publicados entre el 2 de marzo y el 30 de agosto de 1897. Entre el 20 de mayo y el 30 de agosto de 1897 leer a Jaurès era pan comido ya que escribía en cuatro publicaciones a la vez: La Dépêche, La Petite République, Le Matin y La Lanterne. Un año más tarde, en 1898, colaboró de manera ocasional en Cosmopolis y en La Revue de Paris, con esta última mantendrá su colaboración hasta 1910. 2650 artículos y actas de sus intervenciones aparecieron en l'Humanité en los diez años que van desde el inicio del periódico, el 18 de abril de 1904, hasta el día de su asesinato, el 31 de julio de 1914. Además, durante este período, Jaurès redacta un artículo de opinión, primero quincenal y después cada tres semanas, en la Revue de l'enseignement primaire et primaire supérieure (Silvestre, 2010 ; Rioux, 2005 ; Rebérioux, 1994).

Una escritura tan prolífica no puede pasar desapercibida, sobre todo si tenemos en cuenta que utiliza la reflexión sobre la prensa y su rol de creadora de opinión como argumento en muchos de los artículos de nuestro corpus. $Y$ es que podemos afirmar sin miedo a equivocarnos que Jean Jaurès es ante todo un periodista magnífico o, como dice Madeleine Rebérioux (1994, p. 44), Jaurès en su práctica cotidiana es más periodista que intelectual.

El contexto en el que desarrolla su práctica periodística también es trascendental. La Tercera República conoce el "triunfo, definitivo y masivo, de la prensa popular". Según Thérenthy et Vaillant (2004, p. 14), durante este período (1870-1940), "el periodismo está demasiado omnipresente y forma parte integrante de la nueva cultura como para que se pueda pensar, de forma seria, librarse de él". La llegada de las rotativas y de la linotipia transforman los periódicos en un producto rentable hacia 1900 y la ley del 29 de julio de 1881 sobre la libertad de prensa permite su desarrollo. Poco a poco, la morfología de los periódicos cambia e introducen fotos, ilustraciones, publicidad, etc., al mismo tiempo que las redacciones se hacen cada vez más complejas y se 
profesionalizan. Pero, aunque encontramos la misma evolución en todas partes, podemos hablar de un "periodismo a la francesa y su resistencia -relativa- a los modelos anglo-sajones" (Thérenthy, 2007, p. 27) de la misma forma que Jean Jaurès defenderá un "socialismo a la francesa".

Político y periodista son casi sinónimos durante la Tercera República. En el caso de Jaurès estas dos facetas son parte de la misma lucha: la de la República, la de la democracia, la del socialismo y la de la paz, cuatro aspectos de un único objetivo: "reconciliar a todos los pueblos por la universal justicia social" (Jaurès, 18 de abril de 1904). "El periodismo fue el arma secreta de Jaurès. Ya no es posible disociar lo uno de lo otro. Se confunden", resume Emonde Charles-Roux (en Silvestre, 2010, p. 5).

Acabamos de citar una frase del primer artículo de Jean Jaurès en l'Humanité, un artículo esencial para comprender su pensamiento sobre el socialismo y sobre el periodismo. En él comprobamos que la verdad es un eje central de este pensamiento. $\mathrm{Si}$, como dijo a la juventud del instituto de Albi en su célebre discurso de 1903, "el valor es buscar la verdad y decirla, es no sufrir la ley de la mentira triunfante que pasa, y no hacerse eco, con nuestra alma, con nuestra boca y con nuestras manos de los aplausos imbéciles y de los abucheos fanáticos”, ¿qué periodismo se debe ejercer? La respuesta es lógica:

Es a través de informaciones amplias y exactas como nos gustaría dar a todas las inteligencias libres la manera de comprender y de juzgar por ellas mismas los acontecimientos del mundo. La gran causa socialista y proletaria no necesita ni de la mentira ni de medias verdades ni de informaciones tendenciosas ni de noticias forzadas o truncadas ni de procedimientos oblicuos o calumniosos. No necesita que disminuyamos y degrademos injustamente a los adversarios, ni que mutilemos los hechos. Solo las clases en decadencia tienen miedo de toda la verdad: y yo quisiera que la democracia socialista, unida a nosotros de corazón y de pensamiento, esté orgullosa pronto de constatar con nosotros que todos los partidos y todas las clases sociales están obligados a reconocer la fidelidad de nuestras actas, la garantía de nuestras informaciones, la exactitud controlada de nuestras correspondencias. Me atrevo a decir que es así como realmente marcaremos todo nuestro respeto por el proletariado. Así verá, lo espero, que esta preocupación constante y escrupulosa de la verdad, incluso en las batallas más feroces, no debilita el vigor de la lucha: al contrario, da a los golpes contra el prejuicio, la injusticia y la mentira una fuerza decisiva. (Jaurès, 18 de abril de $1904)^{3}$

Es decir, en el viejo debate sobre la objetividad y la honestidad, Jaurès escogió la honestidad: "el autor habla como hombre libre a otros hombres libres, sin ligaduras a intereses personales, sin ni una onza de una propaganda al dictado. Por supuesto, el texto tiene una intención y no la esconde" (Silvestre, 2010, p. 31). Por eso, no es 
casualidad que describa específicamente "nuestro objetivo" en la portada del primer número de l'Humanité.

Cabe destacar que la democracia como centro de la comunicación es uno de los aportes más importantes de Jaurès al oficio. $Y$ es ahí donde debemos comprender l'Humanité, más allá de que sea, también, una herramienta del socialismo, es necesario estudiarlo como lo que es: un periódico. No en vano, toda la experiencia periodística de Jaurès que hemos mencionado al principio de este epígrafe está insertada en el periódico que creó. Coincidimos así plenamente con Silvestre en la idea de que un "periódico no nace jamás de la nada, se inscribe, aunque sea rompiendo, en una historia, la de la prensa. De La Dépêche y de La Petite République, Jaurès heredó lo mejor para hacer el periódico que respondiese a sus deseos por completo al fin" (2010, p. 122). Pero Jean Jaurès no solo heredó la historia de la prensa sino que supo escribir una nueva página:

Apasionado, Jaurès sentó las bases de un periodismo comprometido, constantemente renovado en función de la actualidad; comentó las evoluciones del país insistiendo regularmente sobre los valores que eran los suyos y que intentó difundir en el seno de la SFIO y más allá. A diferencia de la de sus discursos, su escritura periodística es a la vez sencilla y elevada, a menudo cincelada brillantemente, usando a veces la ironía, pero nunca el sarcasmo o la desinformación. El Jaurès periodista intenta sobre todo convencer. (Kermoal, 2014b, p.5)

Acerca del Jaurès periodista hay un testimonio muy valioso. Se trata de un texto redactado por Victor Snell para Floréal, l'hebdomadaire illustré du monde du travail, publicado el 31 de julio de 1920, seis años después del asesinato del líder del socialismo francés. Snell fue secretario de redacción de l'Humanité desde 1907 hasta 1912. Para Snell, Jaurès era un "gran periodista" aunque "ignoraba -desde el punto de vista técnico (...)- casi todo de un periódico" y "no venía a la redacción nada más que ocasionalmente". Muy preocupado por la verdad, Jean Jaurès advertía cada día a sus compañeros: "Sobre todo nada de injurias". Según Snell, "la cortesía era su principal preocupación" y "también estaba muy atento a los pequeños detalles". "Bondadoso con todos sus colaboradores", les daba a todos su lugar en la redacción. "Avaro en las alabanzas", era prudente y delicado con sus observaciones: "solo, los defectos de forma y la incorrección del lenguaje excitaban su severidad". Por su parte, la erudita jauresiana más prolífica, Madaleine Rebérioux (1994, p. 44), resume de la siguiente forma su manera de trabajar:

En esta "jornada de Jaurès", sobreabundante (...) el artículo destinado a l'Humanité no se redactaba por la mañana, sino, salvo excepción, a finales de la tarde. Normalmente en su casa porque apenas iba al periódico: así que tenía que telefonear su texto, enterarse de lo que el secretario de comunicación había 
recopilado, ordenarle que verificase la cortesía de la forma y su corrección gramatical, etc.

Así pues, al hablar del "Jaurès, periodista", debemos hacer hincapié en su contribución a la historia de la comunicación tanto como pensador como hacedor (y muy prolífico) con un alto sentido ético, preocupado por la verdad y la exactitud de sus informaciones, convirtiendo a la democracia en el eje central de su manera de entender y practicar el oficio.

\section{EL COMBATE POR LA PAZ DE JEAN JAURÈS: ANÁLISIS DE SUS TEXTOS EN L'HUMANITÉ}

Si hacemos una división de géneros periodísticos de la forma más simple y más general posible, existen dos grandes categorías: los géneros informativos y los géneros de opinión. La producción periodística de Jaurès se enmarca en la opinión, aunque él reivindica constantemente la verdad (siendo meticuloso con las fuentes, por ejemplo) y la responsabilidad de los periódicos. Sus artículos en l'Humanité están siempre en la portada, pero se van desplazando hacia un lugar menos preeminente con el devenir de los años. Especialmente en los inicios del periódico, Jaurès escribe los "premiers-Paris": "El 'premier-Paris' es el término designado para calificar el artículo de cabecera de un periódico encargado de tratar la cuestión política del día. Generalmente redactado por el director del periódico, corresponde aproximadamente a nuestro editorial moderno" (Thérenthy, 2007, p. 16). A medida que la opinión de Jaurés ya no tiene ese lugar preeminente es frecuente que sus artículos formen parte de una cobertura más amplia en la que hay otros puntos de vista, informaciones complementarias, fotografías, mapas, etc. Ese es el caso, por ejemplo, de todos los artículos que escribe sobre la "ley de los tres años".

La guerra y las relaciones internacionales están presentes en l'Humanité desde el primer número. Las explicaciones complejas de acontecimientos complejos, a menudo simplificados en otros periódicos de la época, y el cuestionamiento constante de la realidad son los pilares del pensamiento jauresiano en general, y del pensamiento jauresiano de paz, en concreto; y es lo que, como veremos a continuación, encontramos en los artículos de nuestro corpus: un análisis exhaustivo de "las causas y las consecuencias" (incluso titula así uno de sus artículos).

\subsection{República, democracia y socialismo}

Para Silvestre (2010, p. 35), "el periodismo de Jaurès se basa, desde sus primeros años, en una práctica profesional y en una idea política: la república social". Del asunto Dreyfus, Jaurès, que en un principio estaba en contra del capitán acusado de haber entregado documentos secretos a los alemanes y andando el tiempo escribió las "Pruebas" de su inocencia, sale con una certeza: "que la conquista de la República y la 
emancipación del proletariado forman parte de la misma lucha" (Rioux, 2005, p. 125). Por eso, en la manera jauresiana de concebir la República, este término es sinónimo de democracia y los dos son lo mismo que decir socialismo. Jaurés es un amante atrapado por la República (Silvestre, 2010, p.24), un "profeta de la República" incluso (Rebérioux, 1994, p.21) y un "creyente del socialismo" (Fabre, 2014, p. 23), un socialismo que "no puede crecer nada más que en la democracia y en la República, que no puede triunfar nada más que después de una revolución material y moral" (Rioux, 2005, p. 129). O dicho de otra manera más clara, si los republicanos se alían con los reaccionarios contra los obreros, la República se pierde (Silvestre, 2010, p.14), tal y como sucedió en la huelga de mineros de Carmaux con la que nace su militancia socialista, y es exclusivamente en el seno de la República que el socialismo es posible.

Estas tres palabras equivalentes (República, democracia y socialismo) constituyen además el único camino posible para la paz entre los individuos, las clases y los pueblos ya que "a medida que se desarrollan entre los pueblos y los individuos la democracia y la razón, la historia queda exenta de recurrir a la violencia" (Jaurès, 18 de abril de 1904). Por tanto, según defiende el autor: "es por el crecimiento de la democracia y el socialismo, y por ahí exclusivamente, que estos sufrimientos serán apaciguados, que estos problemas dolorosos serán resueltos" (Jaurès, 9 de julio de 1905). Nótese que con la utilización del adverbio "exclusivamente" ("seulement" en el original), Jaurès deja bien clara su posición: la paz solo es posible por la democracia, el socialismo y la República.

Por tanto, en el pensamiento de Jaurès, si el socialismo es sinónimo de paz, la guerra es sinónimo de la situación injusta del proletariado: "La guerra es como la explotación directa del trabajo obrero, una de las formas del capitalismo, y el proletariado puede entablar una lucha sistemática y eficaz contra la guerra, como ha iniciado una lucha sistemática y eficaz contra la explotación de la fuerza obrera" (Jaurès, 9 de julio de 1905), defiende, haciendo a su vez equivalentes la lucha de clases y el combate por la paz.

Pero, ¿de qué hablamos cuando hablamos de democracia? Jaurès lo explicita desde el primer artículo aparecido en l'Humanité el día de su lanzamiento: el sufragio universal, la educación laica y una ley más equitativa. Una definición de democracia que coincide con la del historiador Rémond (1974, p. 9) cuando habla de las diferencias entre esta y el liberalismo: "la democracia es el sufragio universal, el gobierno del pueblo, mientras que el liberalismo es el gobierno de una elite", explica.

$\mathrm{Y}$, ¿de qué hablamos cuando hablamos de socialismo? Jaurès lo describe en "La paz y el socialismo" (9 de julio de 1905): "El socialismo quiere organizar la colectividad humana; pero no es una organización de coacción; es bajo la ley general de justicia y armonía que evitará toda tentativa de explotación, dejará a las naciones la libre 
disposición de ellas mismas en la humanidad y a los individuos la libre disposición de ellos mismos en la nación". En este fragmento vemos claramente que en Jaurès el socialismo se basa en la libertad individual y colectiva y en la justicia.

Jean Jaurès es un filósofo al que le gusta ir hasta la raíz; y eso se nota en que sus textos son más morales que políticos. Para él, si todos los seres humanos (las mujeres incluidas -Jaurès, 1 de diciembre de 1912-) tienen acceso a la verdad y a la oportunidad de practicar la libertad de pensamiento, contribuirán a la civilización y a la paz. Podemos afirmar entonces que Jaurès es un intelectual que aboga por la intelectualidad para todo el mundo, mirándose siempre en el espejo de la Francia revolucionaria y republicana, un espejo que, según Rémond (1974, p. 181), "ha suscitado un nacionalismo democrático" que es la base del pensamiento del fundador de l'Humanité.

Además, la libertad de pensamiento en Jaurès hace alusión a informarse, a preguntarse las cosas, a tener una opinión propia, pero también a no tener miedo de la verdad. Dada la importancia que le da a la libertad de pensamiento, no es de extrañar que sea a esta "inteligencia del pueblo", que enfrenta a las "masas" que ceden a los "pánicos locos", a la que hace un llamamiento en el artículo publicado el día de su asesinato:

Para resistir la prueba, los hombres necesitan nervios de acero o más bien una razón firme, clara y calmada. Es a la inteligencia del pueblo, a su pensamiento, a lo que debemos hacer hoy un llamamiento si queremos que se pueda mantener dueño de sí mismo, reconducir el pánico, dominar la crispación y vigilar la marcha de los hombres y de las cosas, para apartar de la raza humana el horror de la guerra. (Jaurès, 31 de julio de 1914)

Si tenemos en cuenta que Jaurès es un hombre de honor, que incluso se batió en duelo para defenderlo, entenderemos que para él defender la justicia social es, antes que nada, una cuestión de honor, tal y como reivindica, por ejemplo, en este fragmento: "No separaréis la reivindicación proletaria de justicia social de la reivindicación humana de orgullo universal, de dignidad universal: es el honor de la Francia socialista y revolucionaria" (Jaurès, 29 de mayo de 1905).

También está orgulloso de ser francés porque Francia, heredera de la Revolución, tiene un papel que jugar en el proceso de civilización y de paz. Esto hace que algunos autores lo sitúen en el marco del "mesianismo republicano" (Fabre, 2014, p.7). Para Jaurès, la "verdadera fuerza" de Francia es su capacidad de convertirse en una fuerza de paz, incluso si ha cometido "errores", "cuando se decida a ser ella misma, será una de las grandes potencias del mundo, por la justicia y por la razón" (Jaurès, 21 de mayo de 1912). En otras palabras, para Jaurès, Francia está "profundamente anclada en la paz" (Duclert, 2013, p. 17) y eso la convierte en una especie de primus inter pares. Es 
por esta razón que "Jaurès ve en la República y su afirmación en Francia una garantía mayor de la paz general en Europa” (Duclert, 2013, p. 19).

La red de equivalencias se va haciendo cada vez más compleja: Francia, la civilización, la justicia, la democracia y la República son la misma cosa, según la argumentación de los textos de Jaurès, y todo eso es una descripción extremadamente precisa de lo que es el socialismo que propugna.

\subsection{La nación y el internacionalismo}

Jean Jaurès era nacionalista en un momento en el que todo el mundo lo era. El proyecto patriótico de la Tercera República había triunfado gracias a la enseñanza escolar republicana, una cultura militar que idealizaba al soldado y la moral republicana que llamaba al compromiso militar heroico (Lambert, 2014). Como reconoce Rebérioux (1975, p. 33), "la religión de la patria permaneció, hasta finales del siglo XX, como una ideología republicana, en la línea de los jacobinos y de Michelet". En ese contexto, siendo Jaurès un republicano y un demócrata convencido, su militancia patriótica no debe sorprendernos.

Entre los textos analizados, el que aborda esta cuestión de manera más clara es el de "El socialismo y la patria" (Jaurès, 29 de mayo de 1905). Este texto es una transcripción de la intervención del líder de la SFIO durante un debate organizado por el Comité Republicano Socialista de Clignancourt en el que Jaurès confronta sus ideas con las de Gustave Hervé (socialista antimilitarista hasta 1912, cambiará esta militancia por el patriotismo y después derivará hacia el fascismo) a propósito de la discusión entre nación e internacionalismo. En este texto, Jaurès se proclama al mismo tiempo nacionalista e internacionalista y defiende que la combinación de ambas es una garantía de paz.

Después de la huelga de mineros de Carmaux, muchos socialistas criticaron la elección de Jaurès como candidato a la Asamblea nacional porque no era "un verdadero socialista". Y tenían razón en que Jaurès tenía algunas diferencias con el pensamiento marxista tradicional. Quizás su original manera de concebir la relación entre el nacionalismo y el internacionalismo es una de sus disidencias más notables. La máxima marxista de que "los proletarios no tienen patria" era para él un "despropósito pesimista". Frente a esta idea marxista, Jaurès defiende que "los proletarios de todos los grandes países de Europa se sentían incorporados a la vida nacional de cada uno de los grandes países", justificado por su papel en el desarrollo histórico de su nación, bien en la unificación alemana, bien en la Revolución francesa. Esta historia es, para el autor, "el patrimonio del proletariado" y el proletariado "incluso si ha sido despojado, incluso si ha sido robado, tenía sobre este patrimonio histórico un título que se confundía precisamente con la existencia combinada del proletariado y de la patria". Como historiador amateur, Jean Jaurès no estaba dispuesto a aceptar 
que la nación francesa, con su educación republicana, sus conquistas laicas y sus progresos científicos, fuera una propiedad exclusiva de la burguesía. También considera que todas esas experiencias particulares serán claves para una sociedad nueva:

Los proletarios de todos los países, al mismo tiempo que viven en la tradición revolucionaria de su nación, crean y preparan en común una vida revolucionaria universal, una patria nueva más elevada y más vasta que todas las naciones: la patria del trabajo, la nación del derecho, la patria de las reivindicaciones y de las esperanzas socialistas. (Jaurès, 29 de mayo de 1905) ${ }^{6}$

Jaurès confía en la sociedad que vendrá gracias al socialismo, pero mientras llega, "la vida nacional de todos los países está todavía a merced de las clases enemigas" y hay que protegerla. Encontramos aquí de nuevo la crítica al capitalismo como desencadenante de "guerras de odio y asesinato". Al contrario del "pacifismo revolucionario" que defiende Hervé, que es "un pacifismo insurreccional, a la vez antimilitarista y antipatriótico" (Lambert, 2014, p. 4), Jaurès argumenta que aceptar que no se tiene patria es aceptar convertirse en el esclavo de otra nación y si eso ocurre, la gente se concentrará en la liberación nacional y no en los problemas sociales. En resumen, para Jaurès defender la patria es esencial para la defensa de la revolución.

Mientras que para el internacionalismo de la época, "la guerra es también una esperanza porque desencadenaría el proceso revolucionario" (Lambert, 2014, p.4), para Jaurès es una gran catástrofe a evitar a toda costa. El fundador de la Sección Francesa de la Internacional Obrera (SFIO) apuesta por "revoluciones pacíficas" (Jaurès, 23 de enero de 1905) porque el precio de una guerra, que define a lo largo de sus textos como una herida, un ultraje y un deshonor para toda la humanidad, es demasiado elevado.

Para hacer compatible la defensa de la nación y de la paz, Jaurès defiende un cambio radical del ejército: con la transformación de los "ejércitos profesionales y de castas en milicias populares" (Jaurès, 9 de julio de 1905). "Solo el sistema de milicias puede, organizando a todos los ciudadanos válidos de la frontera, organizar una fuerza de cobertura eficaz sin desorganizar la fuerza total de la nación" (Jaurès, 25 de febrero de 1905). Durante el debate de la ley de los tres años ${ }^{7}$, Jaurès defenderá que son las milicias nacionales y no el aumento del servicio militar las que pueden vencer a Alemania en una hipotética guerra de agresión (Jaurès, 11 de marzo de 1913).

\subsection{Las relaciones internacionales nación y el internacionalismo}

Para Jaurès, las relaciones internacionales deben desarrollarse en un marco en el que el derecho, la justicia y la lealtad sean las protagonistas porque "la verdadera fuerza de los Estados ya no está en el orgullo de la conquista y en la brutalidad de la opresión, sino en el respeto a las libertades y al derecho, en la preocupación por la 
justicia y la paz" (Jaurès, 30 de julio de 1914). Como expresa Duclert (2013, p. 78), para Jaurès, "la introducción de la justicia en las relaciones internacionales es la garantía de la paz en el mundo". O, tal y como resume Silvestre, "Jaurès, es el derecho" (2010, p. 10) y "no se contenta con cuestionar, con indignarse, él invoca el derecho" (2010, p. 17).

En esta lógica, "arbitraje" es la palabra que mejor describe la concepción de la naturaleza de las relaciones internacionales que tenía Jaurès. La fe del periodista en este sistema para solventar los conflictos es total. En relación al conflicto marroquí, por ejemplo, afirma: "este método internacional nos parece excelente para prevenir y reducir los conflictos" (Jaurès, 17 de junio de 1905). En otro de los momentos de tensión en el norte de África defiende que "una franca internacionalización de Marruecos disipará para siempre toda posibilidad de conflicto; y será al mismo tiempo un excelente resultado y un buen ejemplo" (Jaurès, 2 de septiembre de 1905). En los numerosos artículos que dedica al enfrentamiento franco-alemán en Marruecos invocará constantemente las bondades de la Conferencia de Algeciras que reunió a los representantes de once países europeos, de Estados Unidos y de Marruecos y que consagró la internacionalización del país magrebí y la posición privilegiada de Francia y España. Su balance de esta Conferencia era muy optimista: "Es una transacción razonable que no hiere a nadie en su amor propio". Algeciras es para este defensor de la paz "un precedente extraordinario" que "pone de manifiesto las virtudes de las deliberaciones internacionales" y, por esa razón, subraya la importancia de la lealtad al tratado porque "a partir de ahora la cuestión marroquí es una cuestión internacional que ha sido solucionada internacionalmente" (Jaurès, 3 de abril de 1906).

El argumento del arbitraje es recurrente en los textos sobre Marruecos, pero aparece también en relación a otros conflictos. Tal es el caso del tratado de Portsmouth entre Japón y Rusia que pone fin a la guerra ruso-japonesa:

La intervención del presidente Roosevelt es una victoria del arbitraje porque su vigorosa acción conciliadora ha estado sostenida por la simpatía de todo el mundo civilizado. No había recibido el mandato de las dos potencias beligerantes; pero se atrevió a actuar casi como si hubiese mandato, y es la consciencia universal, horrorizada por las hecatombes del ayer y del mañana, la que lo ha investido de una autoridad inmensa y eficaz. (...) ¿Qué no podría el conjunto de los pueblos civilizados, no solo para acabar, sino para prevenir los conflictos? El arbitraje internacional, tan ridiculizado por algunos, aparece cada vez más como una institución posible y como una institución necesaria. Hay que desarrollarla. (Jaurès, 17 de junio de 1905) ${ }^{8}$

En este artículo, Jaurès profundiza aún más en la red de equivalencias que hemos analizado en el apartado 3.1 y añade nuevos elementos: "Arbitraje, democracia, internacionalismo proletario, son términos vinculados y solidarios. El que desarrolla y 
fortifica uno, desarrolla y fortifica los otros. Sería un crimen no aprovechar la tregua universal que otorga la paz en Extremo Oriente para preparar las condiciones de una paz humana duradera" (Jaurès, 17 de junio de 1905).

La otra gran preocupación de Jaurès al abordar la cuestión de las relaciones internacionales es la diplomacia, más concretamente, los peligros y los resultados funestos de la diplomacia secreta. Para criticar a la diplomacia francesa, el periodista personifica a Francia y la presenta como una víctima de las elecciones insensatas de sus diplomáticos (17 de junio de 1905; 9 de julio de 1905; 6 de noviembre de 1908; 5 de abril de 1911; 27 de abril de 1911; 8 de mayo de 1911). Y frente a "la diplomacia imprudente, ávida o recelosa de los gobernantes capitalistas y de los gobernantes feudales", opone como ideal la "diplomacia pacífica, abierta, leal del proletariado internacional" (Jaurès, 9 de julio de 1905).

Siguiendo a Fabre (2014, p.13), Jaurès esperaba "que las Grandes Potencias de su época podrían ser democratizadas en el interior y conducidas a entenderse en el exterior" y "es en esta óptica en la que ha esperado poder aprovechar los acercamientos y las alianzas". Si nos centramos más específicamente en las relaciones de Francia con el resto de potencias, encontramos que en los textos analizados el periodista insiste en tres aspectos fundamentales para asegurar la paz: el acercamiento franco-alemán, el triángulo Francia-Inglaterra-Alemania y los acuerdos con Rusia.

El acercamiento franco-alemán será posible, según nuestro autor, gracias a la solidaridad y la unión del proletariado francés y del proletariado alemán que con "su común y firme voluntad" podrán "conquistar la paz por la organización y la emancipación de todos los trabajadores (Jaurès, 9 de julio de 1905). Para el líder socialista francés, la guerra franco-prusiana no podía en ningún caso justificar una guerra de revancha. Aunque las heridas de ese enfrentamiento seguían sangrando en la época de nuestros textos, el autor defiende que "la paz es la condición previa y absoluta de la solución del problema de Alsacia y Lorena". Para eso, establece 4 posibles vías: la devolución de los territorios a Francia, una gran autonomía dentro de Alemania, una participación de los dos pueblos o la autonomía legislativa en el seno de un Parlamento europeo. En todo caso, "esta solución solo es posible por un progreso de la democracia y del espíritu de justicia; y este progreso solo es posible con la certeza de la paz" (Jaurès, 11 de mayo de 1913).

En cuanto al triángulo Francia-Inglaterra-Alemania, para Jaurès las tres son "fuerzas necesarias para la civilización" y "todo conflicto que implique a Alemania, Inglaterra, Francia, o a dos de estos pueblos, sería un desastre para la humanidad" (Jaurès, 9 de julio de 1905). En plena lucha contra la ley de tres años, defiende que "la solución necesaria” para garantizar la paz es un acuerdo entre las tres: “¿por qué Francia, Inglaterra y Alemania no concluirían una entente que sería la garantía de paz del 
mundo? Es la única salida del abismo. Es el único camino de salvación para Europa" (Jaurès, 12 de marzo de 1913).

Según Fabre (2014, p. 13), "desde 1904 con la guerra ruso-japonesa, y sobre todo a partir de 1905, fecha de la primera Revolución rusa, Jaurès advirtió de manera cada vez más fuerte de los peligros de la alianza con Rusia, que podía poner a la Francia republicana a remolque de la política retrógrada y arriesgada del Imperio de los zares". Para el fundador de l'Humanité, hay una relación directa entre los acuerdos con Rusia y la burguesía republicana francesa más reaccionaria. Si "el primer derecho y el primer deber de una nación es vivir", este acuerdo de las elites tenía dos efectos negativos sobre Francia: "agravaba el peso de la reacción francesa" y "la Francia republicana se convertía en Extremo Oriente en la sirviente de las ambiciones y las imprudencias de Rusia" (Jaurès, 9 de julio de 1905).

\subsection{El deber y la responsabilidad de la paz}

Si la guerra es "detestable", "salvaje”, "ciega", atroz", "deplorable”, "amenazadora", "loca”, "estéril", "espeluznante”, "abominable", "terrible”, "maldita”, "espantosa”, en definitiva, un "crimen"; si los hombres que nos gobiernan son "temerarios", "enloquecidos", "cobardes", "vanidosos", "torpes", "culpables", "imprudentes", "malvados", "chauvinistas", "belicosos", "infantiles", "hipócritas", "cómplices", en resumen, "lobos"; entonces solo nos queda el deber de impedir la guerra y de preguntarnos quiénes son los responsables de poner a la civilización en peligro. "Deber" y "responsabilidad" son dos ideas claves para comprender el pensamiento de paz de Jean Jaurès y para aproximarnos a los textos aquí analizados.

Lo esencial para Jaurès es que la guerra es evitable (Fabre, 2014, p.10) y los proletarios tienen el deber de mantener la paz. Defiende al proletariado como una fuerza de paz "constante" siempre en alerta para "vigilar en su génesis los primeros conflictos que, al desarrollarse, podrían producir una guerra". Frente al monstruo de la "guerra permanente y latente", el líder socialista reconoce que "la fuerza obrera no está todavía lo suficientemente organizada", pero puede actuar a favor de la paz "que, para ser eficaz, debería ser internacional” (Jaurès, 9 de julio de 1905).

Si el socialismo, tal y como ya hemos argumentado, es para Jaurès, equivalente a la democracia, el deber ciudadano de impedir la guerra ("Son los buenos ciudadanos de Francia, llevados al abismo por los malvados o por los ingenuos, los que tienen que tomar el control. Deben impedir que la situación que se desarrolla en Marruecos desencadene en un conflicto", 2 de julio de 1911) es un deber socialista.

Pero si en Jaurès el deber es colectivo, la responsabilidad es individual y concreta (nunca conceptual -Rebérioux, 1994, p. 92-) y para exigirla agudiza su pluma contra sus adversarios. Así, por poner algunos ejemplos, en "El zarismo enloquecido" (Jaurès, 15 de octubre de 1904) apunta directamente al zar; después de la 
Conferencia de Algeciras llama la atención sobre la enorme responsabilidad que acaba de adquirir el ministro de Asuntos Exteriores (Jaurès, 3 de abril de 1906); o no lo tiembla el pulso para denunciar "la espantosa y abominable matanza" del general Amade (Jaurès, 18 de abril de 1906).

El diputado del Tarn es especialmente implacable con Théophile Delcassé, ministro francés de Asuntos exteriores entre 1898 y 1905, al que llega a calificar de "el más culpable de los hombres" (Jaurès, 17 de junio de 1905). También es muy duro con la "diplomacia de finanzas" de la que "M.Régnault ha sido el agente apasionado" y "M. Pichon ha sido el servidor ciego o el cómplice". Jaurès escribe esto a propósito de la "política criminal" en Marruecos que permite la llegada al poder del enemigo del sultán, Moulaï-Hadif, y escoge como título una frase que expresa de manera clara y concisa su idea de responsabilidad: "Los crímenes se pagan" (Jaurès, 27 de abril de 1911). Esta idea de la guerra como crimen es una de las que más repite en los artículos analizados.

Sin embargo, responsabilidad individual no es sinónimo de responsabilidad exclusiva. Para Jaurès, la responsabilidad es compartida "entre uno y otro" (24 de abril de 1905) que a menudo son Francia y Alemania:

Igual que consideramos que la diplomacia de M. Delcassé ha sido imprudente, incorrecta o culpable eliminando a Alemania de un acuerdo en el que tenía derechos que reivindicar e intereses serios que defender, igual Alemania tendría mala fe en hacer difíciles los acuerdos complementarios que pueden precisar 0 rectificar el acuerdo franco-inglés. (Jaurès, 24 de abril de 1905) ${ }^{9}$

Tampoco podemos pasar por alto al hablar de la responsabilidad en Jaurès su crítica a los periódicos que no contribuyen a un clima de paz al aplaudir las aventuras guerreras de las potencias contribuyendo a alimentar la retórica belicosa de la derecha nacionalista más reaccionaria (15 de octubre de 1904, 23 de enero de 1905, 5 de abril de 1905, 2 de septiembre de 1905, 3 de abril de 1906, 26 de abril de 1907, 16 de abril de 1908, 6 de noviembre de 1908, 22 de abril de 1911, 27 de abril de 1911, 26 julio de 1911, 21 de mayo de 1912, 25 de enero de 1913, 11 de marzo de 1913).

Ya desde 1912, Jaurès nos invita a mantenernos alerta "siempre" y se adelanta a explicar lo que sucederá en la Primera Guerra Mundial. Las "lecciones siniestras" (Jaurès, 12 de junio de 1913) de los Balcanes nos hablan de la existencia de la "barbarie" que se desencadena que no es ajena a Europa: "En el Occidente europeo se desarrollan las mismas fuerzas crueles y bajas". Como ejemplo de esto último basta el debate en torno a la ley de los tres años.

1913 es el año de la lucha contra esta ley de la que Jaurès se convertirá en "el alma de una gran campaña de masas" (Fabre, 2014, p.16). La primera crítica que hace Jaurès es la incapacidad de esta ley de cumplir con el objetivo militar (Jaurès, 3 de 
marzo de 1913). Francia, con los datos en la mano, no puede ganar una guerra de agresión iniciada por una Alemania con una población altamente superior, la única estrategia útil para la victoria son las milicias populares, de las que ya hemos hablado (Jaurès, 11 de marzo de 1913).

Y si no sirve para cumplir el supuesto objetivo militar, ¿para qué sirve la ley de los tres años? Para nada, según Jaurès (14 de marzo de 1913). Entonces, ¿cómo se pueden solucionar los conflictos? La respuesta de Jaurès es que ante la complejidad de los conflictos hay que "despejar estas situaciones confusas, filtrar esta mezcla desordenada, preparar la solución pacífica de las dificultades, porque cada una de las causas del conflicto, una vez aislada y precisada es más fácil de resolver". Y esta solución es el deber del proletariado internacional (Jaurès, 9 de julio de 1905) que debe utilizar para ello la razón (Jaurès, 6 de noviembre de 1908). La idea es que los proletarios unidos pueden imponer a sus gobiernos la utilización del arbitraje a través de la "huelga general preventiva" (Jaurès, 18 de julio de 1914).

El 30 de julio de 1914, aún escribe Jaurès en l'Humanité que la paz sigue siendo posible porque "en todas partes los socialistas tienen la conciencia de su deber", "el deber de utilizar estos días o estas horas para denunciar el crimen, para afirmar y organizar la solidaridad de los proletarios de todos los países contra la abominable amenaza se redobla para todos nosotros".

El 31 de julio hará un llamamiento a la "sangre fría" y será su último grito desesperado a favor de la paz: "El peligro es grande, pero no es invencible si guardamos la claridad de espíritu, la firmeza de la voluntad, si sabemos al mismo tiempo del heroísmo de la paciencia y del heroísmo de la acción. La vista neta del deber nos dará la fuerza de cumplirlo" (Jaurès, 31 de julio de 1914). Ese mismo día recibió dos tiros a bocajarro en la cabeza en el Café du Croissant y al día siguiente la historia de Europa escribió una de sus páginas más negras.

\section{CONCLUSIONES}

Nadie duda de que Jean Jaurès fue asesinado por su lucha a favor de la paz. Ha quedado patente que creyó hasta su último aliento que la paz era posible y que las ideas que defendió y sus reflexiones siguen siendo válidas un siglo después. Jean Jaurès tenía una capacidad de análisis y de prever la realidad que era única: nadie como él supo ver las consecuencias que tendría un conflicto a escala europea. Pero no le bastaba con anticiparse a las cosas, su objetivo era comprender el mundo para interpretarlo e interpretarlo para transformarlo.

Para hacerlo, siempre iba hasta el fondo en todas las cuestiones, buscando explicar las causas y las consecuencias. El historiador que era le ayudaba en esta búsqueda. Y el filósofo de sus primeros años le imponía una mirada moral, basada en el derecho, la justicia y el honor. 
Si la guerra era un "crimen", y esta imagen la utilizó mucho en sus textos, había que impedirla. Y para impedirla, había que pensarla. Podemos afirmar que Jean Jaurès fue un pensador de la guerra que reflexionó durante toda su vida sobre el papel del ejército en la democracia y sobre todas las cuestiones militares de su época. Prueba de ello es que la guerra estuvo presente en l'Humanité desde el primer número.

Pero, tal y como hemos intentado demostrar aquí, Jaurès también fue un periodista con mayúsculas que contribuyó al oficio de manera admirable y un pensador del periodismo, de su contribución a la democracia, de la alianza de cierta prensa con la derecha más reaccionaria y de su potencial para ser una herramienta de emancipación promoviendo el conocimiento de "toda la verdad". El magnífico orador que era dejó huella en sus textos y podemos incluso hablar de un "estilo Jaurès" en el que abundan la ironía, las metáforas, las preguntas retóricas, las anáforas o la intertextualidad. Implacable con los defectos de forma y las incorrecciones lingüísticas, Jean Jaurès practicó la opinión, pero siempre basada en la información y en fuentes fiables.

En sus textos, República, democracia y socialismo forman un todo de civilización y emancipación, alejándose de las tesis marxistas al defender la complementariedad entre nacionalismo e internacionalismo. También defendía unas relaciones internacionales marcadas por el derecho, la justicia, la lealtad, una diplomacia abierta sin cláusulas secretas y el arbitraje. Por último, encontramos la idea del deber y la responsabilidad de paz como una cuestión transversal en todos los artículos analizados.

Su pensamiento de paz desarrollado en l'Humanité se puede resumir en tres mandamientos que reiteró especialmente en los últimos artículos de su vida: los buenos ciudadanos deben ante todo reflexionar y "desenredar la realidad", los buenos ciudadanos deben permanecer unidos frente a un peligro tan grande y los buenos ciudadanos deben mantener la sangre fría para evitar la catástrofe.

El pensamiento de Jean Jaurès es tan prolífico y tan actual que merece especial atención en estudios posteriores. Algunas líneas a explorar podrían ser el canon literario que desarrolla a través de su alter ego, Le Liseur, la idea del periodismo que comparte con Antonio Gramsci o las relaciones existentes entre las publicaciones l'Humanité, Vorwärts y L'Avanti.

\footnotetext{
Notas

${ }^{1}$ La traducción de todas las citas, originalmente en francés, es nuestra. Reproduciremos solo el original de las citas más importantes.

${ }^{2}$ Fernández Lagunilla, M (1999). La lengua en la comunicación política l: El discurso del poder. Cuadernos de Lengua Española. Madrid: Arco/Libros.

Maingueneau, D. (1986). Éléments de linguistique pour le texte littéraire. Paris: Bordas.

Wodak, R. y Meyer, M (2003). Métodos de análisis crítico del discurso. Barcelona: Gedisa.
} 
${ }^{3}$ " C'est par des informations étendues et exactes que nous voudrions donner à toutes les intelligences libres le moyen de comprendre et de juger elles-mêmes les événements du monde. La grande cause socialiste et prolétarienne n'a besoin ni du mensonge, ni du demimensonge, ni des informations tendancieuses, ni de nouvelles forcées ou tronquées, ni des procédés obliques ou calomnieux. Elle n'a besoin qu'on diminue et rabaisse injustement les adversaires, ni qu'on mutile les faits. II n'y a que les classes en décadence qui ont peur de toute la vérité : et je voudrais que la démocratie socialiste, unie à nous de cœur et de pensée, fût fière bientôt de constater avec nous que tous les partis et toutes les classes sont obligés de reconnaître la loyauté de nos comptes rendus, la sûreté de nos renseignements, l'exactitude contrôlée de nos correspondances. J'ose dire que c'est par là vraiment que nous marquerons tout notre respect pour le prolétariat. II verra bien, je l'espère, que ce souci constant et scrupuleux de la vérité, même dans les plus âpres batailles, n'émousse pas la vigueur du combat : il donne au contraire aux coups portés contre le préjugé, l'injustice et le mensonge une force décisive."

${ }^{4}$ Teniendo en cuenta que el "editorial" francés, a diferencia del español, aparece firmado como un artículo de opinión más.

${ }^{5}$ " Pour résister à l'épreuve, il faut aux hommes des nerfs d'acier ou plutôt il leur faut une raison ferme, claire et calme. C'est à l'intelligence du peuple, c'est à sa pensée que nous devons aujourd'hui faire appel si nous voulons qu'il puisse rester maître de soi, refouler les paniques, dominer les énervements et surveiller la marche des hommes et des choses, pour écarter de la race humaine l'horreur de la guerre »

6 «les prolétaires de tous les pays, en même temps qu'ils vivent dans la tradition révolutionnaire de leur nation, créent et préparent en commun une vie révolutionnaire universelle, une patrie nouvelle plus haute et plus vaste que toutes les nations : la patrie du travail, la nation du droit, la patrie des revendications et des espérances socialistes "

${ }^{7}$ En 1913, el gobierno francés propuso modificar la ley de 1905, que establecía el servicio militar obligatorio de dos años para todos los franceses, por una ley que lo ampliaba a tres, pero que los estudiantes de las universidades y las grandes écoles podían realizar en sus centros de estudio. Esta ley en la práctica suponía que los hijos de las clases más altas no realizaban el servicio militar, acabando así con la democratización que supuso la ley de 1905.

${ }^{8}$ " L'intervention du président Roosevelt c'est une victoire de l'arbitrage ; car sa vigoureuse action conciliatrice a été soutenue par la sympathie de tout le monde civilisé. II n'avait pas reçu mandat des deux puissances belligérantes ; mais il a osé agir presque comme s'il avait mandat, et c'est la conscience universelle, épouvantée des hécatombes de la veille et du lendemain, qui l'avait investi d'une autorité morale immense et efficace. (...) que ne pourrait point l'ensemble des peuples civilisés, non seulement pour terminer, mais pour prévenir les conflits ? L'arbitrage international, si raillé par quelques beaux esprits, apparaît de plus en plus comme une institution possible et comme une institution nécessaire. II faut le développer »

${ }^{9}$ « Or, autant nous estimons que la diplomatie de M. Delcassé a été imprudente, incorrecte ou coupable en éliminant l'Allemagne d'un arrangement où celle-ci avait des droits à revendiquer et de sérieux intérêts à défendre, autant l'Allemagne aurait mauvaise grâce à rendre difficiles les arrangements complémentaires qui peuvent préciser ou rectifier l'accord franco-anglais » 


\section{Corpus utilizado}

Jaurès, J. (18 de abril de 1904). Notre but. L'Humanité. Recuperado de http://gallica.bnf.fr/ark:/12148/bpt6k250186x/f1.item [Consultado el 23 de mayo de 2017]

Jaurès, J. (15 de octubre de 1904). La guerre russo-japonaise. L'Humanité. Recuperado de http://gallica.bnf.fr/ark:/12148/bpt6k250366v.item [Consultado el 4 de agosto de 2017]

Jaurès, J. (23 de enero de 1905). La mort du tsarisme. L'Humanité. Recuperado de http://gallica.bnf.fr/ark:/12148/bpt6k2504665/f1.item [Consultado el 25 de mayo de 2017]

Jaurès, J. (24 de abril de 1905). L'un et l'autre. L'Humanité. Recuperado de http://gallica.bnf.fr/ark:/12148/bpt6k2505564/f1.item [Consultado el 25 de mayo de 2017]

Jaurès, J. (29 de mayo de 1905). Le socialisme et la patrie. L'Humanité. Recuperado de http://gallica.bnf.fr/ark:/12148/bpt6k250591m/f1.item [Consultado el 13 de abril de 2017]

Jaurès, J. (17 de junio de 1905). Par le droit. L'Humanité. Recuperado de http://gallica.bnf.fr/ark:/12148/bpt6k250610s/f1.item [Consultado el 25 de mayo de 2017]

Jaurès, J. (9 de julio de 1905). La paix et le socialisme. L'Humanité. Recuperado de http://gallica.bnf.fr/ark:/12148/bpt6k2506327/f1.item [Consultado el 4 de agosto de 2017]

Jaurès, J. (2 de septiembre de 1905). La question marocaine. L'Humanité. Recuperado de http://gallica.bnf.fr/ark:/12148/bpt6k250687v.item [Consultado el 25 de mayo de 2017]

Jaurès, J. (3 de abril de 1906). Après Algesiras. L'Humanité. Recuperado de http://gallica.bnf.fr/ark:/12148/bpt6k250897t/f1.item [Consultado el 25 de mayo de 2017]

Jaurès, J. (26 de abril de 1907). Dans une impasse. L'Humanité. Recuperado de http://gallica.bnf.fr/ark:/12148/bpt6k2512854/f1.item [Consultado el 25 de mayo de 2017]

Jaurès, J. (2 de abril de 1908). Barbarie stérile. L'Humanité. Recuperado de http://gallica.bnf.fr/ark:/12148/bpt6k2516361/f1.item [Consultado el 4 de agosto de 2017]

Jaurès, J. (16 de abril de 1908). L'aveu. L'Humanité. Recuperado de http://gallica.bnf.fr/ark:/12148/bpt6k2516361/f1.item [Consultado el 25 de mayo de 2017]

Jaurès, J. (21 de abril de 1908). Prétendus démentis. Nouveaux aveux. L'Humanité. Recuperado de http://gallica.bnf.fr/ark:/12148/bpt6k251641g.item [Consultado el 25 de mayo de 2017]

Jaurès, J. (26 de agosto de 1908). Acculé. L'Humanité. Recuperado de http://gallica.bnf.fr/ark:/12148/bpt6k2517650/f1.item [Consultado el 25 de mayo de 2017]

Jaurès, J. (6 de noviembre de 1908). Détente nécessaire. L'Humanité. Recuperado de http://gallica.bnf.fr/ark:/12148/bpt6k2518324.item [Consultado el 25 de mayo de 2017]

Jaurès, J. (11 de febrero de 1909). Les causes et les conséquences. L'Humanité. Recuperado 
de http://gallica.bnf.fr/ark:/12148/bpt6k251929r.item [Consultado el 25 de mayo de 2017]

Jaurès, J. (7 de mayo de 1909). Massacres arméniennes. L'Humanité. Recuperado de http://gallica.bnf.fr/ark:/12148/bpt6k252013m/f1.item [Consultado el 4 de agosto de 2017]

Jaurès, J. (5 de abril de 1911). Au Maroc. L'Humanité. Recuperado de http://gallica.bnf.fr/ark:/12148/bpt6k252707z.item [Consultado el 25 de mayo de 2017]

Jaurès, J. (22 de abril de 1911). Casse-cou. L'Humanité. Recuperado de http://gallica.bnf.fr/ark:/12148/bpt6k252724h.item [Consultado el 25 de mayo de 2017]

Jaurès, J. (24 de abril de 1911). Le coup est fait. L'Humanité. Recuperado de http://gallica.bnf.fr/ark:/12148/bpt6k2527268/f1.item [Consultado el 25 de mayo de 2017]

Jaurès, J. (27 de abril de 1911). Les crimes se paient. L'Humanité. Recuperado de http://gallica.bnf.fr/ark:/12148/bpt6k252729d.item [Consultado el 25 de mayo de 2017]

Jaurès, J. (3 de mayo de 1911). Assez de folies. L'Humanité. Recuperado de http://gallica.bnf.fr/ark:/12148/bpt6k2527357/f1.item [Consultado el 25 de mayo de 2017]

Jaurès, J. (8 de mayo de 1911). Conséquences fatales. L'Humanité. Recuperado de http://gallica.bnf.fr/ark:/12148/bpt6k252740p/f1.item [Consultado el 25 de mayo de 2017]

Jaurès, J. (2 de julio de 1911). L'inévitable. L'Humanité. Recuperado de http://gallica.bnf.fr/ark:/12148/bpt6k2527959/f1.item [Consultado el 25 de mayo de 2017]

Jaurès, J. (26 de julio de 1911). Compensations. L'Humanité. Recuperado de http://gallica.bnf.fr/ark:/12148/bpt6k252819c/f1.item [Consultado el 25 de mayo de 2017]

Jaurès, J. (4 de noviembre de 1911). L'Accord Franco-Allemand. L'Humanité. Recuperado de http://gallica.bnf.fr/ark:/12148/bpt6k252920m/f1.item [Consultado el 25 de mayo de 2017]

Jaurès, J. (21 de mayo de 1912). Pas à ce point. L'Humanité. Recuperado de http://gallica.bnf.fr/ark:/12148/bpt6k253116b/f1.item [Consultado el 25 de mayo de 2017]

Jaurès, J. (25 de noviembre de 1912). Grève préventive. L'Humanité. Recuperado de http://gallica.bnf.fr/ark:/12148/bpt6k253297s/f1.item [Consultado el 25 de mayo de 2017]

Jaurès, J. (1 de diciembre de 1912). Les femmes et la guerre. L'Humanité. Recuperado de http://gallica.bnf.fr/ark:/12148/bpt6k253303g/f1.item [Consultado el 4 de agosto de 2017]

Jaurès, J. (3 de diciembre de 1912). Incertitudes. L'Humanité. Recuperado de http://gallica.bnf.fr/ark:/12148/bpt6k2533057/f1.item [Consultado el 4 de agosto de 2017]

Jaurès, J. (25 de febrero de 1913). Dans une impasse. L'Humanité. Recuperado de 
http://gallica.bnf.fr/ark:/12148/bpt6k253390f/f1.item [Consultado el 25 de mayo de 2017]

Jaurès, J. (3 de marzo de 1913). La couverture. L'Humanité. Recuperado de http://gallica.bnf.fr/ark:/12148/bpt6k253396q.item [Consultado el 25 de mayo de 2017]

Jaurès, J. (10 de marzo de 1913). L'Avertissement. L'Humanité. Recuperado de http://gallica.bnf.fr/ark:/12148/bpt6k253402d/f1.item [Consultado el 25 de mayo de 2017]

Jaurès, J. (11 de marzo de 1913). Démasqués. L'Humanité. Recuperado de http://gallica.bnf.fr/ark:/12148/bpt6k253403s.item [Consultado el 25 de mayo de 2017]

Jaurès, J. (12 de marzo de 1913). La solution nécessaire. L'Humanité. Recuperado de http://gallica.bnf.fr/ark:/12148/bpt6k2534045.item [Consultado el 25 de mayo de 2017]

Jaurès, J. (14 de marzo de 1913). Frivolité funeste. L'Humanité. Recuperado de http://gallica.bnf.fr/ark:/12148/bpt6k253406x/f1.item [Consultado el 25 de mayo de 2017]

Jaurès, J. (16 de marzo de 1913). L'armement du peuple. L'Humanité. Recuperado de http://gallica.bnf.fr/ark:/12148/bpt6k253408p/f1.item [Consultado el 25 de mayo de 2017]

Jaurès, J. (18 de marzo de 1913). Symptomes. L'Humanité. Recuperado de http://gallica.bnf.fr/ark:/12148/bpt6k2534100/f1.item [Consultado el 25 de mayo de 2017]

Jaurès, J. (31 de marzo 1913). Mise au point. L'Humanité. Recuperado de http://gallica.bnf.fr/ark:/12148/bpt6k253423g/f1.item [Consultado el 25 de mayo de 2017]

Jaurès, J. (11 de mayo de 1913). L'Alsace-Lorraine et Berne. L'Humanité. Recuperado de http://gallica.bnf.fr/ark:/12148/bpt6k2534647.item [Consultado el 4 de agosto de 2017]

Jaurès, J. (12 de mayo de 1913). Sinistres leçons. L'Humanité. Recuperado de http://gallica.bnf.fr/ark:/12148/bpt6k253495n/f1.item [Consultado el 4 de agosto de 2017]

Jaurès, J. (10 de julio de 1913). Criminelle incohérence. L'Humanité. Recuperado de http://gallica.bnf.fr/ark:/12148/bpt6k253523s.item [Consultado el 4 de agosto de 2017]

Jaurès, J. (18 de julio de 1914). Les furieux. L'Humanité. Recuperado de http://gallica.bnf.fr/ark:/12148/bpt6k253888p.item [Consultado el 4 de agosto de 2017]

Jaurès, J. (27 de julio de 1914). Une lueur d'espoir. L'Humanité. Recuperado de http://gallica.bnf.fr/ark:/12148/bpt6k253897n/f1.item [Consultado el 12 abril de 2017]

Jaurès, J. (30 de julio 1914). L'action. L'Humanité. Recuperado de http://gallica.bnf.fr/ark:/12148/bpt6k2539006/f1.item [Consultado el 12 abril de 2017]

Jaurès, J. (31 de julio de 1914). Sang-froid nécessaire. L'Humanité. Recuperado de http://gallica.bnf.fr/ark:/12148/bpt6k253901k.item [Consultado el 12 abril de 2017]

\section{Referencias}

Candar, G. (2009). La gauche coloniale en France. Socialistes et radicaux (1885-1905). En Mil neuf cent. Revue d'histoire intellectuelle (27, (1), pp. 37-56). 
Duclert, V. (2013). Jean Jaurès. Combattre la guerre, penser la guerre. Paris: Éditions Fondation Jean Jaurès.

Fabre, R. (2014). Le pacifisme de Jaurès entre patriotisme et internationalisme. Conferencia celebrada en Toulouse el 17 de mayo de 2014. Recuperado de http://www.grepmp.com/wp-content/uploads/2016/10/24-Fabre-Jaur\%C3\%A8sReluRFfinal.pdf

Fabre, R. (2015). Jaurès et le Maroc. Un long combat pour la paix et le droit des peuples. Paris: Éditions Fondation Jean Jaurès.

Kermoal, B. (2014). Jaurès: réformer l'armée, agir pour la paix». Note $n^{\circ} 2$. Fondation Jean Jaurès. Recuperado de https://jean-jaures.org/nos-productions/jaures-reformer-l-armeeagir-pour-la-paix

Kermoal, B. (2014b). Jaurès journaliste. Note $n^{0} 10$. Fondation Jean Jaurès. Recuperado de https://jean-jaures.org/nos-productions/jaures-journaliste

Kermoal, B. (2014c). Jaurès et l'attentat de Sarajevo. Note $n^{\circ} 24$. Fondation Jean Jaurès. Recuperado de https://jean-jaures.org/nos-productions/jaures-et-l-attentat-de-sarajevo

Kermoal, B. (2014d). Jaurès et les dernières tentatives de paix. Note $\mathrm{n}^{\circ} 28$. Fondation Jean Jaurès. Recuperado de https://jean-jaures.org/nos-productions/jaures-et-les-dernierestentatives-de-paix

Lambert, A-S. (2014). L'opinion publique française en 1914 entre pacifisme et bellicisme. BNF. Recuperado de http://classes.bnf.fr/rendezvous/pdf/ete14_pacifisme.pdf

Marcobelli, E. (2013). La France de 1914 était-elle antimilitariste? Les socialistes et la Loi de trois ans. Paris: Éditions Fondation Jean Jaurès.

Rebérioux, M. (1975). La République radicale? 1898-1914. Collection: Nouvelle histoire de la France contemporaine, 11. Paris: Éditions du Seuil.

Rebérioux, M. (1994). Jaurès. La parole et l'acte. Paris: Découvertes Gallimard.

Rémond, R. (1974). Le XIXe siècle. 1815-1914. Collection: Introduction à l'histoire de notre temps, 2. Paris: Éditions du Seuil.

Rioux, J-P. (2005). Jean Jaurès. Paris: Perrin.

Snell, V. (31 juillet 1920). Jaurès à son journal. En Floréal, l'hebdomadaire illustré du monde du travail $(26$, p. 606).

Thérenty, M-Ė. y Vaillant, A. (2004). Presse et plumes : journalisme et littérature au XIXe siècle. Paris: Nouveau monde.

Thérenty, M-Ë. (2007). La littérature au quotidien. Poétiques journalistiques au XIXe siècle. Paris: Éditions du Seuil.

Silvestre, C. (2010). Jaurès, la passion du journaliste. Paris: Le temps des cerises. 